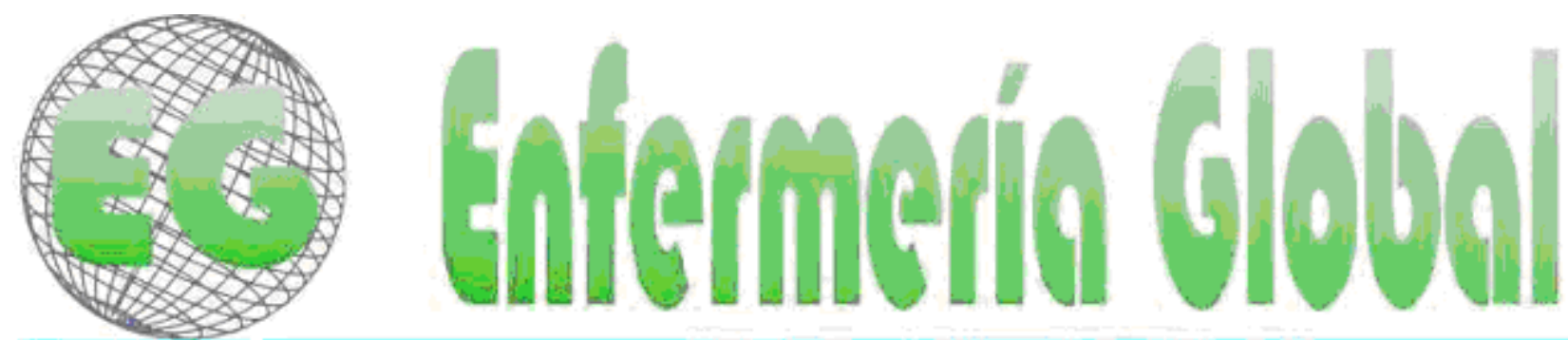

15SN 1696-6*\$S

Revista electrônica cuatrimestral de Enfermeria

$N^{\circ} 18$

Febrero 2010

www.um.es/egloball

CLÍNIGA

\title{
EDUCACIÓN EN DIABETES CON DEFICIENTES VISUALES: UNA EXPERIENCIA UNIVERSITARIA
}

\section{EDUCAÇ̃̃O EM DIABETES COM DEFICIENTES VISUAIS: UMA EXPÊRIÊNCIA UNIVERSITÁRIA}

\begin{abstract}
*Pagliuca, LMF., "*Macêdo, KNF., "*Silva, GRF da, ***Cardoso, MVLML
*Profesora Titular del Departamento de Enfermería. Coordinadora del Proyecto de Pesquisa e Extensão Saúde Ocular/CNPq. **Mestres. Alumnas de Doctorado del Programa de Pos-Graduación en Enfermería. Participantes del Proyecto de Pesquisa e Extensão Saúde Ocular como bolsistas da CAPES. ***Profesora Adjunta del Departamento de Enfermería. Universidad Federal de Ceará. Brasil
\end{abstract}

(Trabajo desarrollado en el Proyecto Saúde Ocular/CNPq)

Palabras clave: Ciego, Diabetes, Prevención

Palavras chaves: Cego, Diabetes, Prevenção

\section{RESUMEN}

Relato sobre la experiencia desarrollada con deficientes visuales en la Asociación de Ciegos del Estado de Ceará, en Fortaleza. Se realizó una conferencia para 40 personas, en la que se debatió sobre el concepto, los factores de riesgo y el tratamiento de diabetes, seguida de selección junto con entrevista, verificación de medidas antropométricas y glicemia capilar. En esta fase participaron 26 personas. Los valores que se obtuvieron con relación a glicemia capilar por la cinta variaron de 74 a $193 \mathrm{mg} / \mathrm{dl}$. El índice de edad abarcó el intervalo entre los 11 a 57 años, 17 del sexo femenino y 9 del masculino. El horario de la última comida varió entre 2 y 6 horas. Entre los factores de riesgo que se abordaron, 20 personas se declararon sedentarias; 10 con historia de diabetes en la familia; 4 presentaron peso superior a lo normal y 2 hipertensos. La conclusión a que se llega es que es de fundamental importancia promover y prevenir la diabetes con este tipo de pacientes.

\section{RESUMO}

Relato de experiência desenvolvida com deficientes visuais na Associação dos Cegos do Estado do Ceará, em Fortaleza. Realizou-se uma palestra para 40 pessoas na qual se abordou conceito, fatores de risco e tratamento do diabetes, seguida de triagem, com entrevista, verificação de medidas antropométricas e glicemia capilar. Desta fase, 
participaram 26 pessoas. Os valores obtidos de glicemia capilar pela fita variaram de 74 a $193 \mathrm{mg} / \mathrm{dl}$. A faixa etária abrangeu o intervalo de 11 a 57 anos, 17 do sexo feminino e 9 do masculino. O horário da última refeição variou de 2 a 6 horas. Sobre fatores de riscos abordados, 20 indivíduos alegaram ser sedentários; $10 \mathrm{com}$ história de diabetes na família; quatro com peso acima da normalidade e dois hipertensos. Pode-se concluir que a promoção e prevenção do diabetes são de fundamental importância para essa clientela.

\section{ABSTRACT}

Experience developed with visually impaired people in the Association for the Blind in the city of Fortaleza, Ceará (Brazil). A conference for 40 people was held about the concept, risk factors and treatment of diabetes followed by selection, interview, and verification of anthropometrics measures and capillary glycemia. In this phase 26 people participated. The values obtained related to capillary glycemia by strip varied from 74 to $193 \mathrm{mg} / \mathrm{dl}$. Age index was from 11 to 57 years with 17 females and 9 males. The time of the most recent meal varied between 2 and 6 hours. Among risk factors faced: 20 people reported being sedentary, 10 with a history of diabetes in the family; 4 being overweight y 2 with hypertension. The conclusion we arrive at is that it is of fundamental importance to inform and prevent diabetes in this type of patients.

\section{INTRODUCCIÓN}

Al igual que la diabetes, gran parte de las patologías que afligen al hombre pueden ser evitadas o mantenidas bajo control con acciones de prevención y promoción a la salud. El principal problema de esa patología es su clasificación como silenciosa, o sea, asintomática, cuyo tratamiento inadecuado provoca descontrol metabólico capaz de proporcionar surgimiento de complicaciones que afectan la calidad de vida de las personas portadoras de la diabetes y de los respectivos familiares.

Así, los profesionales de salud deben aumentar las acciones preventivas, con vistas a controlar la glucemia de los pacientes con diagnóstico confirmado y estar atentos a la identificación de las personas con riesgo para desarrollar la enfermedad.

La atención de enfermería abarca no solamente cuidados a los enfermos. También incluye orientaciones de personas e intervención directa en la prevención de enfermedades ${ }^{(1)}$. La simple transmisión de informaciones, en esa situación, puede cambiar hábitos y comportamientos.

Diabetes mellitus abarca un grupo heterogéneo de causas y manifestaciones clínicas, que tienen como denominador común el aumento de glucosa en la sangre. Ello resulta, la mayoría de las veces, por la disminución o producción alterada de insulina por el páncreas, ocasionando modificaciones en el metabolismo de proteínas, grasas, sales minerales y, principalmente, de glucosa $^{(2)}$.

Las consecuencias de la DM a largo plazo incluyen daños en el organismo, disfunción y fallo de varios órganos, especialmente riñones, ojos, nervios, corazón y vasos sanguíneos. Con frecuencia, los síntomas clásicos (pérdida no explicada de peso, polidipsia y poliuria) quedan ausentes, pero puede darse hiperglucemia en grado suficiente para causar alteraciones funcionales o patológicas por largo período antes de que sea definido el diagnóstico. Antes de la aparición de hiperglucemia, acompañada del cuadro clínico clásico de la DM, el 
síndrome diabético pasa por un estadio de alteración del metabolismo de la glucosa, caracterizado por valores glucémicos situados entre la normalidad y la diabética ${ }^{(3)}$.

Actualmente, la clasificación adoptada incluye los estadios clínicos de la DM, desde la normalidad, pasando por tolerancia disminuida a la glucosa y/o glucemia en ayunas alterada hasta la DM propiamente dicha.

Existen dos tipos de diabetes. La del tipo 1 es más frecuente en jóvenes, y requiere uso de inyecciones diarias de insulina para compensar la falta o cantidad insuficiente producida por el cuerpo. El tipo 2, por otro lado, se caracteriza por el funcionamiento inadecuado de la insulina producida por el cuerpo. Este tipo incide con mayor frecuencia en adultos, y es más común en personas con exceso de peso y con antecedentes familiares de diabetes. Su control es realizado como sigue: balanceo de los alimentos ingeridos, práctica de ejercicios físicos, alcance del peso adecuado y, en algunos casos, uso de medicamentos ${ }^{(3)}$.

Los procedimientos diagnósticos usados abarcan la medida de glucosa en suero o plasma tras ayuno de 8 a 12 horas y el test estandarizado de tolerancia a la glucosa (TTG) tras administración de 75 gramos de glucosa anhidra por vía oral, con medidas de glucosa en suero o plasma 0 y 120 minutos después de la ingestión ${ }^{(3)}$.

Al ser práctica y no invasiva, la prueba de glucemia capilar permite controlar los valores glucémicos con indicación, y es realizada mediante el uso de una gota de sangre de la punta del dedo, tras asepsia y punción con aguja apropiada. Esa pequeña gota es colocada en tira especial e introducida en un aparato para verificar los valores ${ }^{(4)}$.

Una vez diagnosticada la diabetes, su portador deberá insertarse en un tratamiento cuyo objetivo es mantener la tasa de glucosa en valores normales o próximos al normal. Ese tratamiento incluye, en principio, la utilización de insulina o hipoglucemiante oral, dieta balanceada y práctica regular de ejercicios físicos. Es a partir de esta rutina cuando se originan los problemas específicos de la clientela portadora de diabetes, principalmente la de bajo nivel socio-económico-cultural ${ }^{(5)}$.

Cualquier persona puede desarrollar la enfermedad. Este grupo abarca al deficiente visual que, con alteración de su percepción sensorial, necesita que los profesionales de enfermería empeñen acciones más específicas destinadas a promover y prevenir la diabetes. Sin embargo, debido a las condiciones de acceso y del modo convencional como las informaciones son repasadas, los deficientes visuales pueden ser perjudicados. Esta realidad llevó al interés en realizar este trabajo, con objeto de establecer contacto directo con esa clientela y proponer acciones de educación en salud.

Para que la educación en salud tenga éxito es preciso considerar las características del público albo esclérotico. En este caso, deficientes visuales, con su nivel de escolaridad, y socioeconómico, entre otros. Los profesionales de salud, como responsables de las orientaciones sobre la salud de los deficientes, deben trabajar para preservar el derecho del deficiente visual a la ciudadanía. Por lo tanto, es papel del enfermero proponer alternativas para el autocuidado.

La salud es un proceso dinámico, instable y se muestra insertado en un contexto ligado a innúmeros factores. Como la relación salud y enfermedad debe ser considerada simbiótica, ningún programa de educación en salud podrá ser llevado a cabo de modo efectivo, eficaz y eficiente sin fundamentos en enfoque educativo, dinámico y adecuado ${ }^{(6)}$. 
Con miras al desarrollo de acciones educativas específicas, fue realizado este estudio con objeto de desarrollar acciones de educación en salud con deficientes visuales, tratando de la diabetes.

\section{MATERIAL Y MÉTODOS}

Este estudio se configura como un relato de experiencia, desarrollado en un proyecto de extensión comunitaria, fundamentado en el pensamiento científico según el cual los métodos son los instrumentos básicos que ordenan los pensamientos en sistemas, trazando el modo de proceder del científico a lo largo de una investigación para alcanzar un objetivo predeterminado $^{(7)}$.

El trabajo resulta del contacto con deficientes visuales, en la perspectiva del alumno de pregrado en enfermería. Este contacto ocurrió con participantes de una asociación de deficientes visuales ubicada en Fortaleza, estado de Ceará, Brasil, durante actividades de educación en salud tratando de la diabetes. Se presenta con característica de extensión comunitaria, ya que asista a personas y les presta cuidados.

\section{RESULTADOS}

Para relatar la experiencia vivida, inicialmente será enfocado el proyecto de investigación y extensión que propició el desarrollo de este estudio, a saber: el Proyecto Salud Ocular.

\section{El proyecto salud ocular}

Como parte de las acciones del Departamento de Enfermería de la Facultad de Farmacia, Odontología y Enfermería de la Universidad Federal de Ceará, el Proyecto Salud Ocular está vinculado a las Pro-Rectorías de Investigación y Extensión y al Consejo Nacional de Desarrollo Científico y Tecnológico - CNPq. En él participan alumnos de pregrado y postgrado que vienen desarrollando actividades de atención e investigación referentes a la promoción de la salud ocular en las etapas del ciclo vital, además de la atención de enfermería al deficiente visual.

Las acciones de educación en salud para los deficientes visuales ya desarrolladas tratan de los siguientes temas: los métodos contraceptivos ${ }^{(8)}$, la prevención y el tratamiento de emergencias domésticas ${ }^{(9)}$ y la hipertensión ${ }^{(10)}$.

Para la exploración táctil, la elaboración de estos materiales y métodos consideró algunos principios como: textura, que deriva de la calidad física del plano, tiene relación directa con el material usado y sugiere el sentido de lo táctil; espacio, que es el área física a ser trabajada, y masa, que es determinada por la cantidad de material utilizado ${ }^{(11)}$.

De modo general, como muestran los estudios promovidos por el Proyecto Salud Ocular, los deficientes visuales se sienten satisfechos con la creación de materiales destinados a ellos en la educación en salud. Se debe destacar que la creación de esas nuevas acciones de enfermería aplicadas en la educación y en la salud con esas personas son de fácil obtención.

A continuación serán expuestas la planificación, ejecución y evaluación de la experiencia vivida con los deficientes visuales. 


\section{Planificación}

El Proyecto Salud Ocular tiene como uno de los campos de investigación y atención los miembros de una asociación de deficientes visuales. En esta institución, los asociados estudian y participan de actividades recreativas. De estas, la más común es el juego de dominó. Anualmente, durante el mes de septiembre, cuando es conmemorada la Semana Social del Ciego, son desarrolladas actividades educativas, como ponencias, encuentros y discusiones sobre varias temáticas. Entre ellas asuntos referentes a la salud.

Por iniciativa de la dirección, los integrantes del Proyecto Salud Ocular fueron invitados a participar de ese evento durante tres días mediante la presentación de temas relevantes en el área de salud.

El tema sugerido por los integrantes del proyecto trató de la prevención de la diabetes. Para ello, fue elaborado un plan de actividades sometido a la dirección de la asociación, y fueron realizadas visitas a la institución para preparar el trabajo de campo.

\section{Ejecución}

Con la supervisión y orientación de la coordinadora del proyecto, nueve becarios desarrollan actividades con los asociados, un público constituido básicamente por adolescentes y adultos.

El trabajo de campo ocurrió en dos momentos. El primero fue una ponencia sobre DM administrada por una estudiante de enfermería, discutiendo concepto, factores de riesgo y tratamiento. El segundo momento, de acuerdo con la invitación, abarcó la consulta de enfermería, con duración de tres días, incluyendo el control de las medidas antropométricas y glicemia capilar, además de preguntas sobre los factores de riesgo para la diabetes. La participación de los deficientes fue voluntaria, sin restricción de sexo o edad.

De las personas que participaron de la ponencia sobre diabetes, 26 asociados se sometieron a la consulta de enfermería, 17 individuos del sexo femenino y nueve del sexo masculino.

Para la realización de la glicemia capilar durante la consulta de enfermería, se preguntó sobre el horario de la última comida, que varió de dos a seis horas. Dos horas después de la ingestión de $75 \mathrm{~g}$ de glucosa, las personas normales tienen glucemia inferior a $140 \mathrm{mg} / \mathrm{dl}$, mientras las diabéticas tienen glucemia igual o superior a $200 \mathrm{mg} / \mathrm{dl}$ y las que presentan intolerancia a glucosa oral valores entre 140 y $200 \mathrm{mg} / \mathrm{dl}^{(2)}$.

De las 26 personas sometidas al test de glucemia capilar, dos presentaron niveles glucémicos alterados. Una de ellas, en el grupo de edad de 51 años o más, relató ser diabética desde hace quince años. La edad de la otra era de sólo 23 años. La diabetes tipo 1, también llamado diabetes infantil-juvenil, incide más en niños y jóvenes, mientras la diabetes tipo 2, también llamada diabetes del adulto o de la madurez, ocurre principalmente en adultos en el grupo de edad de 30 años o más ${ }^{(12)}$. Los niveles de glucemia capilar obtenidos por la cinta variaron de 60 a $193 \mathrm{mg} / \mathrm{dl}$. En estas personas, 24 de los valores estaban por debajo de $140 \mathrm{mg} / \mathrm{dl}$ y dos fueron superiores.

Valores arriba de $140 \mathrm{mg} / \mathrm{dl}$ de glucosa en la sangre son considerados alterados, si son obtenidos al menos dos horas después de la última comida. Existen algunas situaciones que favorecen la aparición de diabetes. Entre ellas destacan: enfermedades coronarias, historia familiar de DM, edad > 45 años, peso $\left(\mathrm{IMC}>25 \mathrm{~kg} / \mathrm{m}^{2}\right)$, sedentarismo, DM gestacional 
previa, uso de medicamentos hiperglucémicos (por ejemplo, corticosteroides, tiazídicos, betabloqueadores, estrógenos) ${ }^{(1)}$.

La promoción de salud dirigida a esa patología se centra en el control y la prevención de los factores de riesgo. En esa clientela, tales factores son considerados relevantes, a pesar de la baja cantidad de personas con alteración de la glucemia capilar.

Conforme observado, el sedentarismo es común entre deficientes visuales. De los participantes, 20 informaron que no practicaban ningún tipo de actividad física. El sedentarismo es la principal causa del aumento de la incidencia de varias enfermedades, entre ellas, hipertensión arterial, diabetes, obesidad, ansiedad, aumento del colesterol, infarto de miocardio. Es considerado el principal factor de riesgo de muerte súbita, estando, la mayoría de las veces, asociado directa o indirectamente a las causas o al agravamiento de la mayoría de las enfermedades ${ }^{(13)}$.

La práctica de ejercicios físicos regulares es indispensable y siempre debe ser enfatizada pues además de actuar como un ítem benéfico para la prevención de la diabetes, proporciona y preserva el bienestar físico, psíquico y social de los individuos. Para los deficientes visuales, la práctica de ejercicios físicos es difícil, ya que no existen normalmente incentivos para ellol, ni locales adecuados y de fácil acceso.

De las personas consultadas, 10 presentaron diabetes mellitus en sus históricos familiares, esto es, existían casos diagnosticados en las familias. La diabetes mellitus tipo 1 tiene poca influencia hereditaria, mientras la diabetes tipo 2 indica fuerte factor hereditario (14). De acuerdo con la Sociedad Brasileña de Diabetes ${ }^{(3)}$, personas que tienen parientes en primer grado con historia de diabetes mellitus son consideradas de riesgo con relación a la diabetes tipo 2.

Cuatro de los deficientes visuales presentaron peso por encima de los patrones de normalidad, o sea, el índice de masa corporal era mayor a $25 \mathrm{~kg} / \mathrm{m}^{2}$. Una importante orientación se refiere al cambio de hábitos alimentarios y a la práctica de ejercicios físicos, factor ya mencionado, capaz de ayudar en la reducción de peso de esas personas y de evitar problemas futuros.

El tratamiento de la diabetes incluye algunos requisitos como modificación del estilo de vida, que abarca la suspensión del tabaquismo, la práctica regular de actividad física, la reducción de los hábitos alimentarios y, en caso necesario, el uso de medicamentos ${ }^{(3)}$.

También se sabe que la práctica de ejercicios reduce la obesidad corporal total, además de la obesidad abdominal, con consecuente disminución de los riesgos de enfermedad cardiovascular y de la diabetes tipo $2^{(15)}$.

Con relación a la hipertensión arterial, en dos de los deficientes visuales fue diagnosticada la enfermedad y en 24 fueron verificados valores dentro de los límites de normalidad.

Enfermedades multifactoriales, como las cardiovasculares, son influenciadas por diversas variables genéticas y ambientales. Conforme a lo divulgado en numerosos estudios, las familias que generalmente conviven en el mismo ambiente sociocultural, pasan a sus descendientes el estilo de vida y los patrones de comportamiento que favorecen el desarrollo de la enfermedad cardiovascular, como hábitos alimentarios inadecuados, sedentarismo y tabaquismo. La relación entre el histórico familiar de enfermedad coronaria y la presencia de factores de riesgo cardiovasculares es cada vez más evidente en varios estudios 
epidemiológicos. De los factores familiares que predisponen a la enfermedad cardiovascular, las dislipidemias están entre las más investigadas y son uno de los principales factores de riesgo para enfermedad cardiovascular en pacientes diabéticos ${ }^{(3)}$. La contribución genética en la determinación de la variabilidad del perfil lipídico es de aproximadamente $60 \%{ }^{(16)}$.

La hipertensión arterial presenta algunas particularidades en los tipos de diabetes. En el tipo 2, la hipertensión forma parte del cuadro del síndrome metabólico, estando presente en casi el $50 \%$ de los clientes en el momento del diagnóstico de la diabetes mellitus. En la diabetes tipo 1, la hipertensión arterial se manifiesta tardíamente, asociada a la microalbuminuria, y permanece más intensa y frecuente en la medida que evolucione para las fases de macroproteinuria y reducción de la función renal ${ }^{(3)}$.

Sin embargo, el ejercicio físico puede contribuir a la reducción del riesgo substancial de enfermedad cardiovascular que afrontan los individuos con diabetes tipo 2 e hipertensión arterial. Existen evidencias de que esos ejercicios pueden mejorar la función vasodilatadora endotelial en individuos en estas circunstancias ${ }^{(15)}$.

\section{Evaluación de la experiencia}

Después de la ponencia desarrollada y las consultas de enfermería, los participantes expresaron la importancia de la actuación de la atención de enfermería al deficiente visual. Durante las consultas, relataron haber comprendido la importancia del diagnóstico precoz, las complicaciones, y también el tratamiento. La dirección de la institución agradeció la actuación a los integrantes del Proyecto Salud Ocular durante la semana social del ciego y elogió el trabajo de campo.

Al final de cada día, la experiencia fue evaluada informalmente entre los alumnos de pregrado y los deficientes visuales. La evaluación fue consolidada en una segunda reunión entre los integrantes del proyecto, cuando evaluaron el plan de actividades inicialmente propuesto. Fueron expuestas verbalmente las dificultades, las facilidades, las estrategias utilizadas, además de nuevas sugerencias levantadas en acta. Sin embargo, el desarrollo de ese trabajo no fue suficiente para provocar cambio de conducta con relación a hábitos saludables para la prevención de la diabetes. Mientras tanto, fue posible sensibilizar a los deficientes para la enfermedad, factores de riesgo y tratamiento.

\section{CONCLUSIONES}

Conforme la experiencia permite inferir, a pesar del número reducido de personas con alteraciones en su glucemia capilar, las acciones de prevención y promoción para los deficientes visuales son esenciales, porque están predispuestos al desarrollo de la diabetes mellitus, debido a los siguientes factores de riesgo identificados: sedentarismo, exceso de peso y antecedente familiar de diabetes e hipertensión arterial.

Se cree que ese tipo de experiencia favorece futuras intervenciones dirigidas a la educación en salud, con aproximación de los factores de riesgo para evitar el desarrollo de la diabetes, y también de enfermedades asociadas. Convivir con personas portadoras de deficiencia es una experiencia incomparable; nos ayuda en nuestro propio desarrollo como futuros profesionales y nos hace pensar sobre la necesidad de estrategias orientadas a la educación en salud con esa clientela, explorando sus sentidos remanentes. 


\section{REFERENCIAS BIBLIOGRÁFICAS}

1 Daniel LF. A enfermagem planejada. 3. ed. São Paulo: E.P.U.; 1981. 13p.

2 Costa AA; Almeida Neto JS. Manual de diabetes. São Paulo: Sarvier; 2000. 127p.

3 Sociedade Brasileira de Diabetes. Consenso brasileiro sobre diabetes. Diagnóstico e classificação do diabetes mellitus e tratamento do diabetes mellitus tipo 2. Versão final e definitiva. Maio de 2002.

4 Smeltzer S; Bare BG.Brunner \& Suddarth. Tratado de enfermagem médico-cirúrgica. 10 ed. Rio de Janeiro: Guanabara Koogan; 2005, v. 1, p.34-43.

5 Damasceno MMC. Os caminhos e descaminhos da assistência aos diabéticos. In: Damasceno MMC; Loureiro MFF; Fernandes AFC. Transtornos vitais no fim do século XX: diabetes mellitus, distúrbios cardiovasculares, câncer, AIDS, tuberculose e hanseníase. Fortaleza: Fundação Cearense de Pesquisa e Cultura; 1999. p.11-14.

6 Campos JQ; Fernandez JAR et al. Administração de saúde e pesquisa de campo. São Paulo: Jotacê; 1997. 78p.

7 Cianciarullo TI apud Ferrari AT. Metodologia da ciência. 3. ed. Rio de Janeiro: Kennedy; 1974.

8 Pagliuca LMF; Rodrigues ML. Métodos contraceptivos comportamentais: tecnologia educativa para deficientes visuais. Rev. Gaúcha Enfermagem, v. 19, n. 2, p. 147-153, 1998.

9 Pagliuca LMF et al. Desenvolvendo tecnologia para prevenção e tratamento de emergências domésticas para cegos. Rev. Bras. Enfermagem, Brasília, v. 49, n. 1, p. 83104, jan./mar.1996.

10 Pagliuca LMF; Macêdo KNF; Silva GRF. Material tátil para a prevenção de hipertensão em deficientes visuais. Rev. Rene, Fortaleza-CE, v. 4, n. 2, p. 75-81, 2003.

11 Pagliuca LMF. A arte da comunicação na ponta dos dedos - a pessoa cega. Revista Latino-americana de Enfermagem, Ribeirão Preto, v. 4, n. esp, p. 127-137, 1996.

12 Costa AA; Neto JS. Manual de diabetes: alimentação, medicação e exercícios. 4. ed. rev. ampl. autor. São Paulo: Sarvier; 2004.

13 Craven RF; Hirnle CJ. Fundamentos de Enfermagem: saúde e funções humanas. Rio de Janeiro: Guanabara Koogan ; 2006.

14 Brasil. Ministério da Saúde. Secretaria de Políticas de Saúde. Departamento de Ações Programáticas e Estratégicas. Manual de hipertensão arterial e diabetes mellitus. Brasília; 2002. 102p.

15 Arsa GL; Lima L; Almeida SMS; Campbell C; Simões H. Diabetes Mellitus tipo 2: Aspectos fisiológicos, genéticos e formas de exercício físico para seu controle. Revista Brasileira de Cineantropometria e Desempenho Humano [Online], Volume 11 Número 1, 2009

16 Fernandes RV. Fatores de risco para doenças cardíacas na infância e adolescência. Disponible en: <http://www.acm.org.br/saude/pediatria doencascardiacas.htm>. Acceso en: 22 jan. 2009.

ISSN 1695-6141

@ COPYRIGHT Servicio de Publicaciones - Universidad de Murcia 